•技术与方法・

\title{
外来物种入侵大数据采集方法的建立与应用
}

邱荣洲 ${ }^{1}$, 赵健 ${ }^{02}$, 陈宏 ${ }^{2}$, 冼晓青 ${ }^{3}$, 池美香 ${ }^{1}$, 翁启勇 $1^{*}$

1. 福建省农业科学院植物保护研究所/福建省作物有害生物监测与治理重点实验室, 福州 350013; 2. 福建省农业科学院数字农业研究所, 福州 350003; 3. 中国农业科学院植物保护研究所, 北京 100193

摘要: 为准确、快速地获取入侵生物野外调查数据, 我们基于全球卫星导航系统、地理信息系统、移动互联网等现代信息技 术提出了外来物种入侵大数据采集方法, 设计并研发了数据表单可自定义的野外调查工具软件一一云采集。该系统以Android 手机为数据采集终端, 采用 C\#和Java语言设计开发, 运用卫星导航定位技术实现野外调查发生位置的快速采集, 通过定义9 种调查指标的数据类型及指标(列值)默认值、图像拍摄、语音录入、排序等 4 个辅助属性，建立调查指标与手机客户端数据录 入界面的关联，实现用户界面可定制的数据录入模式。该系统在国家重点研发项目、福建省科技重大专项及福建省红火蚁 (Solenopsis invicta) 疫情普查等项目的调查任务中予以应用。实践检验表明: 该系统实现了野外调查数据的离线采集、数据同 步、数据查询与输出管理, 将移动智能终端采集取代传统的纸笔记录, 简化了野外调查的流程, 提高了入侵生物野外调查的 数据质量，为外来生物入侵野外调查大数据采集提供了信息化支持。

关键词: 入侵生物; 移动终端; 数据采集; 云采集; 大数据

邱荣洲, 赵健, 陈宏, 洗晓青, 池美香, 翁启勇 (2021) 外来物种入侵大数据采集方法的建立与应用. 生物多样性, 29, 1377-1385. doi: 10.17520/biods.2021030.

Qiu RZ, Zhao J, Chen H, Xian XQ, Chi MX, Weng QY (2021) Research and application of a big data collection method for invasive species surveys. Biodiversity Science, 29, 1377-1385. doi: 10.17520/biods.2021030.

\section{Research and application of a big data collection method for invasive species surveys}

Rongzhou Qiu ${ }^{\circledR 1}$, Jian Zhao ${ }^{\circledR 2}$, Hong Chen², Xiaoqing Xian $^{3}$, Meixiang Chi $^{1}$, Qiyong Weng ${ }^{*}$

1 Institute of Plant Protection, Fujian Academy of Agricultural Sciences/Fujian Key Laboratory for Monitoring and Integrated Management of Crop Pests, Fuzhou 350013

2 Institute of Digital Agriculture Research, Fujian Academy of Agriculture Sciences, Fuzhou 350003

3 Institute of Plant Protection, Chinese Academy of Agricultural Sciences, Beijing 100193

\section{ABSTRACT}

Aims: To quickly and accurately obtain field survey data on invasive species diffusion, we integrated the global navigation satellite system, geographic information system, and mobile internet to design a customizable mobile terminal for the collection of survey data for invasive species. This is important because traditional methods are cumbersome, slow, and error-prone. Additionally, previously designed computational survey methods are designed to address specific survey tasks. Here, we developed a customizable software program, the Yuncaiji data acquisitor, to address these issues.

Method: This system was designed using the C\# and Java languages. It uses navigation satellite positioning technology to help acquire precise location data more rapidly. This method tries to combine navigation satellite positioning with the Android mobile terminal to help acquire precise location data.

Results: This method defines 9 kinds of data and 4 auxiliary attributes, such as default value of column value (indicator), image shooting, voice input, column sorting, establishing the association between data types and user interface, and realized customizable data entry interface.

Conclusion: The Yuncaiji data acquisitor has been successfully tested in several large-scale projects, including key

收稿日期: 2021-01-20; 接受日期: 2021-06-11

基金项目: 国家重点研发项目(2016YFC202105)、福建省科技重大专项(2017NZ0003-1)和福建省属公益类科研院所基本科研专项(2018R1025-4)

* 通讯作者 Author for correspondence. E-mail: wengqy@faas.cn 
national (Chinese) research and development projects, major science and technology projects for the Fujian Province, and for Solenopsis invicta epidemic census. Results show that the Yuncaiji data acquisitor efficiently supports field survey data collection, data synchronization, data exporting, and data management. This system is superior to traditional survey methods involving pen and paper, because the data recoding is easily performed on a smartphone, simplifying data entry and reducing user error. The quality of the invasive species diffusion field data recorded by the Yuncaiji data acquisitor is high, indicating that our system provides suitable computational support for large-scale surveys of the diffusion of invasive species.

Key words: invasive species; mobile terminal; data collection; Yuncaiji data acquisitor; big data

生物入侵已对入侵区的生态环境、社会经济和 人类健康造成严重威胁, 成为 21 世纪五大全球性环 境问题之一(Millennium Ecosystem Assessment, 2005)。入侵生物的传播和扩散是其产生危害的最重 要过程, 通过对入侵物种的本底调查, 可为生物入 侵的管理与控制提供依据(万方浩等, 2002; 鞠瑞亭 等, 2012)。入侵生物调查大数据来源大体分为人工 实地观测和传感器自动记录两种(戴圣骐和赵斌, 2016)。传感器自动记录受制于传感器技术的革新, 目前主要以温、光、湿、气等环境参数和声音、图 像、视频采集为主, 而入侵生物传播和扩散的影响 因子复杂, 调查过程还无法通过机器全自动采集完 成。因此人工实地直接观测仍然是目前最主要的调 查方式。

传统的人工实地观测通过 “全球卫星导航系统 + 地图” 导航到样本位置(陈涁, 2012; 陈宏等, 2018), 人工填写纸质表格并通过相机采集图像数 据, 调查结束后经全面检查验收, 再把数据输入 计算机。这种方法存在以下缺陷: 野外纸质表格填 写工作量较大且不容易定位; 调查过程存在数据 采集规范不足、纸质采集记录丢失、记录数据不完 整、内业数据录入工作量大、数据出错概率高、维 护困难等问题。随着全球卫星导航系统 (global navigation satellite system, GNSS)、地理信息系统 (geographic information system, GIS)、移动互联网、 智能手机等技术的快速发展, 在科学研究、野外调 查、生态环境监测、病虫害监测等专业领域的移动 应用开发也进入快速发展阶段。周园春等(2008)开 发了基于Windows Mobile的野外科考数据采集系 统, 并在青海湖鸟类动态数据调查中进行应用; 申文明等(2013)和邱荣洲等(2014)分别开发了基于 Android平台的生态环境和武夷山生物多样性的移 动数据采集系统, 为野外考察提供了便携的数据 采集工具; 罗卿权等(2011)和赵庆展等(2015)分别
开发了基于GNSS和GIS的有害生物信息采集系统, 实现了病虫害发生位置及属性信息快速采集与查 询; Vankadavath等(2009)开发了植物表型数据采集 系统, 实现了利用掌上电脑 (personal digital assistant, PDA)对实验室和田间大量植物的表型数 据进行统一编目管理; Köhl和Gremmels (2015)开 发了应用于植物育种和遗传的表型数据输入和管 理的移动终端软件; 黄国锐等(2019)开发了一种基 于嵌入式微处理器手持式数据采集终端, 有效地 解决了野外条件下采集数据的实时传输和安全性 问题。由众多非专业志愿者利用手机采集的众源地 理数据 (单杰等, 2014), 如 OpenStreetMap 、 Wikimapia、Foursquare、微博、微信等, 能够记录 各种各样的兴趣点位置、时间和语义信息(潘晓等, 2020), 但是缺乏数据完整性, 一般需要经过处理 才能形成规范的地理信息, 难以满足专业的地理 数据要求(Goodchild, 2007)。由于外来物种调查数 据的采集涉及到其传入、定殖、扩散、危害等各个 阶段, 上述数据采集系统大多数应用在特定的专 业领域, 无法满足入侵生物野外调查需求。

本文以科技部基础调查专项“中国外来物种及 其安全性考察”项目考察成果为基础, 结合国家重 点研发项目对农田、森林、保护区、口岸等不同生 态系统进行本底调研和数据分析的具体需求, 建立 表单可自定义的外来物种入侵大数据采集方法, 开 发了农林科学数据云采集平台(简称“云采集”软件), 以期为中国外来入侵物种调查与协作研究提供野 外调查和网络化的管理工具, 为后续外来入侵物种 扩散空间分布、风险评估和防控预案提供具有空间 信息的基础数据支撑。

\section{系统设计}

\section{1 开发工具}

数据采集设备: 本研究选用华为Ascend P7、集 
思宝 A3S 作为系统测试的采集终端, 其中华为 Ascend P7搭载Android 4.4操作系统, 内存2 GB; 集 思宝A3S搭载Android 5.1操作系统, 内存1 GB。两 款设备均支持卫星导航定位、拍照、WiFi/GPRS/4G 无线网络传输功能。

软件开发环境: 本系统整体由Android手机客 户端和web服务器端组成。其中手机终端开发环境 为: JDK (Java development kit) + Eclipse + Android SDK (Software development kit) + ADT (Android development tools); web端的开发环境为 Microsoft Visual Studio .NET 2013, 利用Microsoft SQL Server 2008 构建系统数据库, 以 Microsoft .NET Framework 4.0为集成开发平台, 通过Visual C\#编程 实现。

\section{2 采集流程设计}

采集流程分为采集前准备、采集数据和数据处 理分析3个阶段(图1)。前期准备主要根据入侵生物 扩散本底数据调查的要求, 建立外来入侵病原物、 节肢动物、植物等各种数据的采集规范, 并确认采 集人员信息及是否打印标本标签。数据采集阶段主 要由采集人员根据采集规范进行调查, 并通过“云 采集”手机客户端进行卫星导航定位、拍照及相关 调查数据记录, 然后上传到服务器。最后通过web 管理系统查看和审核数据, 并进行增加、修改、删 除操作, 以及将审核好的数据按查询条件进行输出,
支持XLS、KML格式及关联图片的批量导出。

\section{3 系统功能设计}

\subsection{1 采集标签管理}

基于二维码技术设计入侵生物野外采集标本 标签, 结合“云采集”软件的二维码扫描识别功能, 建立实物标本与标本记录的关联关系。标签的编号 规则为 “省区代号 +4位顺序号”, 如福建采集的 1 号标本记为350001。将采集标签贴在标本的保鲜袋 左上角, 用 “云采集”软件扫描标签上的二维码，记 录采集标本的信息，包括：编号、当前地理位置、 采集人、采集日期、采集地点、生境类型、物种名 称、图片等(图2)。

如果是新发现种或在野外无法判定物种时，可 带回到实验室鉴定，再通过“云采集”定位到该标本 的记录进行相关信息的修改。为保障采集日期、采 集人、采集地点的准确性, 系统不允许对这3项数据 进行修改。

\subsection{2 采集系统设计}

系统整体由web服务器端和手机客户端组成。 服务器端负责采集数据的存储与管理, 手机客户端 实现对野外调查数据的快速录入采集。入侵生物野 外调查有时会在网络中断或无信号环境中进行, 这 就要求移动数据采集系统支持离线数据传输。离线 应用的数据先在移动端临时存储, 数据的更新与服 务器要有良好的同步机制。

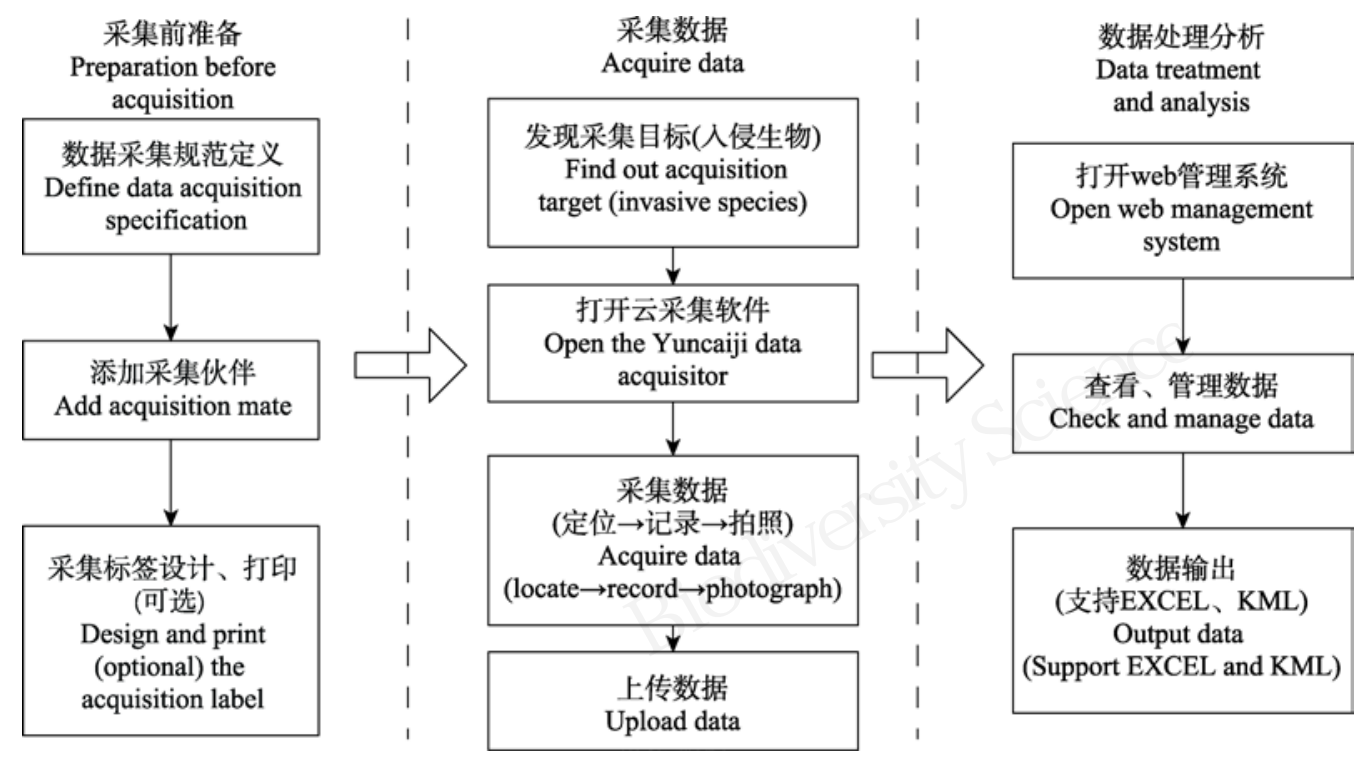

图1 云采集软件外来物种入侵大数据采集工作流程图

Fig. 1 System workflow of big data collection for invasive species survey of the Yuncaiji data acquisitor 


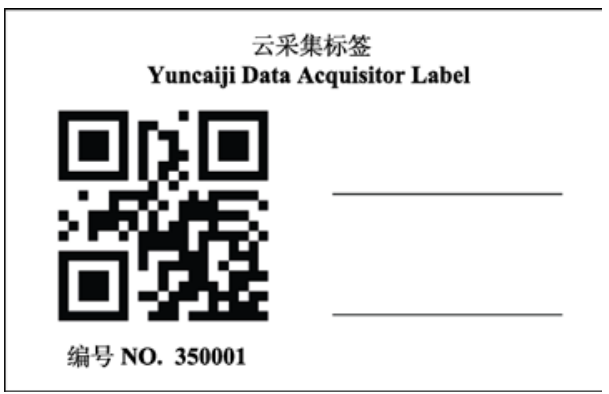

图2 云采集软件采集标签图样

Fig. 2 Sample collection label of the Yuncaiji data acquisitor

(1) Web服务器端功能模块。服务器端应用系统 主要包括采集任务、数据管理、数据接收和系统管 理 4 个模块(附录1)。其中采集任务模块负责数据表 格标准的定制、任务的发起与结束、采集伙伴(任务 参与人)的添加与删除、采集标签的生成与打印等功 能; 数据管理模块实现采集数据的查询、编辑、输 出及地图展示; 数据接收模块通过网络服务方式获 取客户端上传的采集数据文件, 解压后分别存储到 数据库和文件系统; 系统管理模块由用户管理、操 作日志、错误日志、意见反馈等功能组成。

(2)手机客户端软件功能模块。客户端软件主要 包括 4 个模块: 数据采集、数据同步、伙伴动态与系 统设置(附录2)。数据采集模块负责野外数据的录 入、编辑与查看, 以及通过扫描二维码采集标签实 现快速定位数据功能; 数据同步模块通过访问网络 服务实现手机本地采集数据的解析、更新与上传; 伙伴动态模块实现了任务伙伴动态数据的查看和 基于高德地图的采集位置地图浏览功能; 系统设置 模块由软件运行相关的基础功能组成, 包括用户验 证、软件更新、图片大小设置、意见反馈等。

(3)可定制数据采集规范的实现方法。针对外来 物种入侵调查内容指标不确定性问题, 提出数据表 结构可自定义的数据录入模式, 具体实现流程如下: 首先根据入侵生物野外应用的具体需求, 通过web 服务端以任务为单位进行调查指标定制操作。系统 将定制指标先存储到调查任务表中, 然后手机客户 端将任务指标解析成SQLite数据库文件, 最后将存 储在SQLite数据库文件中的数据与后台数据库同步, 将共性指标“采集时间” “采集地点” “采集地地理位 置” “采集人”分别保存到采集数据表的collectTime、 collectaddr、collectGPS、collectUser字段中, 用户定 制差异指标数据保存成固定格式存储到XML数据 类型的xmlcontent字段中。通过将差异调查指标统 一放到一个XML列, 避免了修改数据库表结构, 用 户可根据调查指标的需要对表结构灵活地自定义。

用户界面定制上，通过定义调查指标(字段)的 数据类型, 建立调查指标与手机客户端数据录入界 面的关联, 实现界面可定制的移动数据采集模式。 将系统自定义的调查指标数据类型划分为单行文 本、多行文本、整型数、单精度数、单选项、复选 项、日期、时间和扫码文本共 9 种, 手机客户端根据 数据类型制定相应的约束性内容, 展示不同的录入 界面。调查指标还设计了“列值默认值” “图像拍摄” “语音录入” “排序”等辅助属性作为数据录入界面 的控制器。通过 “列值默认值” 属性自动为部分指标 赋值, 以减少指标值相似或相同引起的重复录入工 作，提高手机录入数据的速度; “排序”属性可设定 各指标在录入界面中的布局顺序; “图像拍摄”和“语 音录入”为“是否显示”的控制属性，一般根据调查 指标选择是否需要开通此功能。

(4)数据同步机制设计。移动终端需要与服务器 同步的数据有调查任务和调查数据两种, 数据同步 模块负责从服务器端下载分配给终端的任务, 通过 与WCF (Windows Communication Foundation, 通讯 开发平台)服务交互实现与服务器的任务列表同步, 以及对本地任务的查看。数据上传子模块负责将采 集数据先缓存到移动端, 当无线网络通畅时再使用 在线模式与服务器实时同步更新。由于图片文件数 据量较大, 所以选择将数据文件整体打包后上传的 方式同步, 以提升数据传输的效率。同步机制如图3 所示。用户登录手机客户端后, 先检查远程服务器 端的WCF数据服务接口是否处于开启状态; 然后 查看是否有本地任务: 如果不存在, 尝试连接WCF 服务下载任务与数据模板, 并存入本地数据库, 然 后显示任务列表项; 如果存在, 则直接显示任务列 表项。在上传数据时, 调查者可以自行选择网络连 接的方式, 或根据Android操作系统的设置自动连 接网络。系统会自动弹出是否成功上传的提示框。

\section{系统实现}

\section{1 位置信息的获取}

通过百度地图Android定位SDK实现移动终端 地理坐标数据的读取。考虑入侵生物的调查采集一 般在野外, 以及高精度定位需求, 采用SDK的设备 


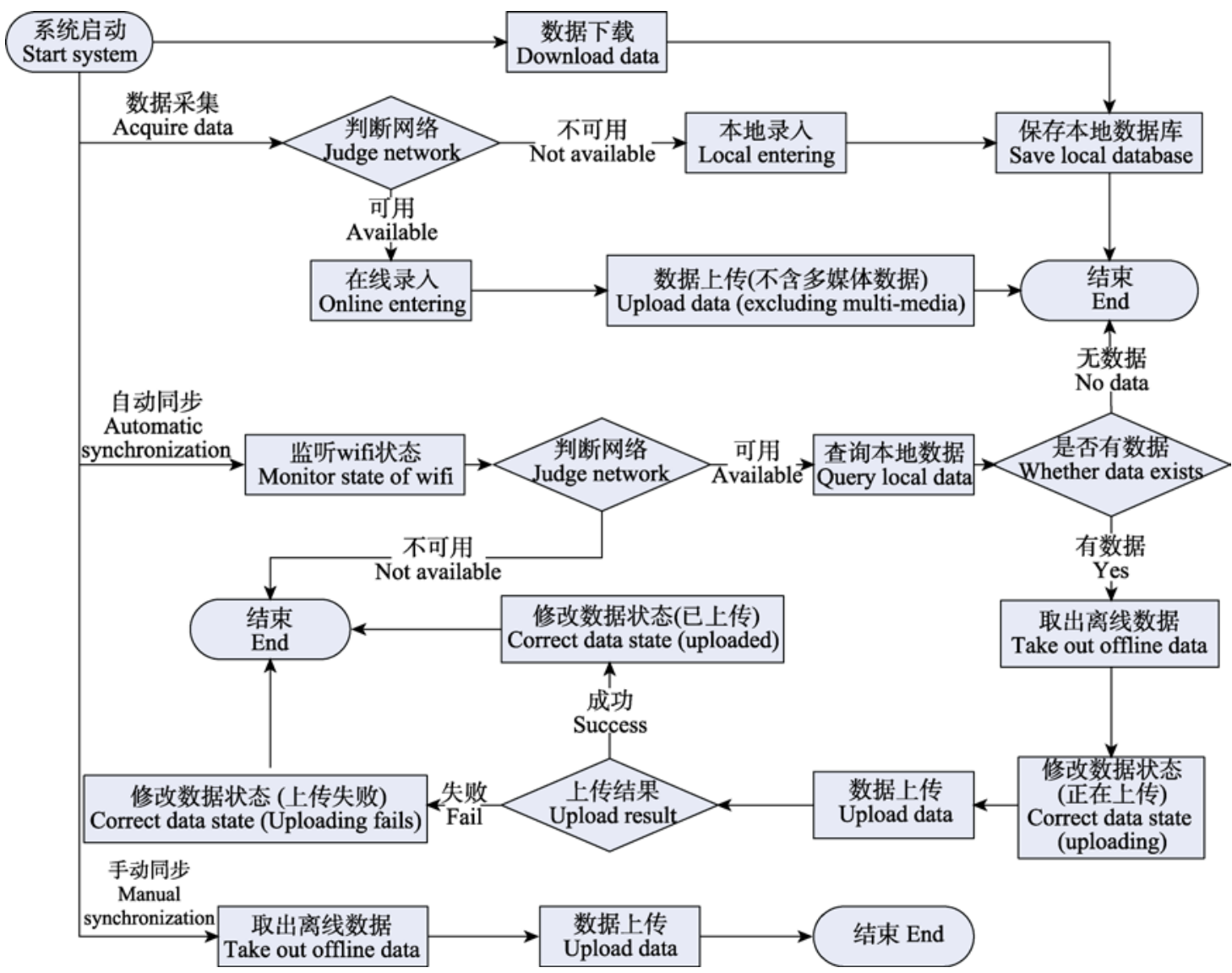

图3 云采集软件数据同步机制

Fig. 3 Data synchronization flowchart of the Yuncaiji data acquisitor

定位模式进行定位。在地理坐标获取成功后, 系统 通过连接百度LBS反地址解析服务获取当前位置所 在的行政区划及详细地址(如“ $x \times$ 省 $x \times$ 县+详细地 址”, 其中详细地址用户可修改)。为测试云采集软 件位置信息的可靠性, 分别在华为Ascend P7智能 手机、集思宝A3S专业卫星导航定位仪上安装云采 集手机客户端软件进行定位精度比较。2016年6月 17日, 在福州国家森林公园采集的18个位置点进行 比较试验, 通过谷歌地球(Google Earth) 比对, 识别 并确认采集点的实际位置, 结果显示(表1), 手机的 平均精度为 $6.02 \mathrm{~m}$, 专业卫星导航定位终端的平均 精度是 $2.40 \mathrm{~m}$, 两台设备的位置精度均小于 $15 \mathrm{~m}$, 可以满足入侵生物物种空间分布、风险评估、防控 预案等数据处理对位置精度的要求。

\section{2 任务创建与数据采集}

根据调查的任务不同，通过web管理端定义不 同的数据采集规范, 移动终端数据采集模块会分别 调用相应的人机交互界面。登录web管理端, 进入发
起任务页面(图4A), 通过设置任务表格的表单自定 义功能(图4B), 可以增加数据列, 设置列名、数据类 型、列值默认值、图像拍摄、语音录入、排序等属 性，快速完成数据采集规范的设定。其中采集时间、 采集地点、采集地地理位置、采集人为固定列，用 户不可修改。登录手机客户端后, 首先将用户权限 范围的调查任务和服务器最新数据同步到本地, 然 后进入任务列表页面，单击某个任务名称进入采集 数据列表页面, 再通过点击右上方或正下方的“+” 号进入数据采集页面(图4C)。数据采集包括当前地 理坐标获取，采集人、采集时间的自动生成及文字、 图像、声音、条码识别等调查采集项的录入。采集 项根据用户定制的采集规范，系统自动调用文本 框、单选下拉菜单、下拉复选框菜单、时间选择控 件、数字键盘、二维码扫描、拍照、录音等相应的 输入方式, 调查人员只要对数据项进行确认就可以 完成大部分的数据采集工作, 减少了文字输入, 提 高数据采集的效率。 
表1 云采集软件位置信息获取功能测试结果

Table 1 Test of location acquisition accuracy using the Yuncaiji data acquisitor

\begin{tabular}{|c|c|c|c|c|c|c|c|c|}
\hline \multirow[t]{2}{*}{$\begin{array}{l}\text { 序号 } \\
\text { No. }\end{array}$} & \multicolumn{2}{|c|}{$\begin{array}{l}\text { 实际位置 } \\
\text { Actual location }\end{array}$} & \multicolumn{3}{|c|}{$\begin{array}{l}\text { 集思宝A3S获取位置 } \\
\text { Location captured by Jisibao A3S smartphone }\end{array}$} & \multicolumn{3}{|c|}{$\begin{array}{l}\text { 华为P7获取位置 } \\
\text { Location captured by Huawei P7 smartphone }\end{array}$} \\
\hline & $\begin{array}{l}\text { 纬度 } \\
\text { Latitude (N) }\end{array}$ & $\begin{array}{l}\text { 经度 } \\
\text { Longitude (E) }\end{array}$ & $\begin{array}{l}\text { 纬度 } \\
\text { Latitude }(\mathrm{N})\end{array}$ & $\begin{array}{l}\text { 经度 } \\
\text { Longitude (E) }\end{array}$ & $\begin{array}{l}\text { 误差 } \\
\text { Error (m) }\end{array}$ & $\begin{array}{l}\text { 纬度 } \\
\text { Latitude }(\mathrm{N})\end{array}$ & $\begin{array}{l}\text { 经度 } \\
\text { Longitude (E) }\end{array}$ & $\begin{array}{l}\text { 误差 } \\
\text { Error (m) }\end{array}$ \\
\hline 1 & $26.15056^{\circ}$ & $119.2889^{\circ}$ & $26.15058^{\circ}$ & $119.2889^{\circ}$ & 2.67 & $26.15062^{\circ}$ & $119.2889^{\circ}$ & 6.12 \\
\hline 2 & $26.15003^{\circ}$ & $119.2877^{\circ}$ & $26.15004^{\circ}$ & $119.2877^{\circ}$ & 1.93 & $26.15003^{\circ}$ & $119.2878^{\circ}$ & 0.39 \\
\hline 3 & $26.15014^{\circ}$ & $119.2876^{\circ}$ & $26.15015^{\circ}$ & $119.2876^{\circ}$ & 1.21 & $26.15012^{\circ}$ & $119.2876^{\circ}$ & 1.94 \\
\hline 4 & $26.14998^{\circ}$ & $119.2876^{\circ}$ & $26.14999^{\circ}$ & $119.2876^{\circ}$ & 1.02 & $26.14996^{\circ}$ & $119.2876^{\circ}$ & 2.62 \\
\hline 5 & $26.15007^{\circ}$ & $119.2874^{\circ}$ & $26.15007^{\circ}$ & $119.2874^{\circ}$ & 0.39 & $26.15003^{\circ}$ & $119.2874^{\circ}$ & 4.64 \\
\hline 6 & $26.15021^{\circ}$ & $119.2873^{\circ}$ & $26.15024^{\circ}$ & $119.2873^{\circ}$ & 3.12 & $26.15020^{\circ}$ & $119.2873^{\circ}$ & 0.89 \\
\hline 7 & $26.15026^{\circ}$ & $119.2872^{\circ}$ & $26.15027^{\circ}$ & $119.2872^{\circ}$ & 1.22 & $26.15023^{\circ}$ & $119.2872^{\circ}$ & 3.34 \\
\hline 8 & $26.15085^{\circ}$ & $119.2865^{\circ}$ & $26.15087^{\circ}$ & $119.2865^{\circ}$ & 1.87 & $26.15087^{\circ}$ & $119.2864^{\circ}$ & 10.54 \\
\hline 9 & $26.15091^{\circ}$ & $119.2864^{\circ}$ & $26.15093^{\circ}$ & $119.2864^{\circ}$ & 1.56 & $26.15097^{\circ}$ & $119.2864^{\circ}$ & 7.49 \\
\hline 10 & $26.15089^{\circ}$ & $119.2862^{\circ}$ & $26.15093^{\circ}$ & $119.2862^{\circ}$ & 4.22 & $26.15095^{\circ}$ & $119.2862^{\circ}$ & 9.65 \\
\hline 11 & $26.15093^{\circ}$ & $119.2859^{\circ}$ & $26.15098^{\circ}$ & $119.2859^{\circ}$ & 5.35 & $26.15085^{\circ}$ & $119.2859^{\circ}$ & 8.70 \\
\hline 12 & $26.15098^{\circ}$ & $119.2856^{\circ}$ & $26.15101^{\circ}$ & $119.2856^{\circ}$ & 3.33 & $26.15097^{\circ}$ & $119.2856^{\circ}$ & 3.64 \\
\hline 13 & $26.14976^{\circ}$ & $119.2852^{\circ}$ & $26.14978^{\circ}$ & $119.2852^{\circ}$ & 1.84 & $26.14971^{\circ}$ & $119.2852^{\circ}$ & 6.16 \\
\hline 14 & $26.14814^{\circ}$ & $119.2853^{\circ}$ & $26.14816^{\circ}$ & $119.2853^{\circ}$ & 1.82 & $26.14824^{\circ}$ & $119.2853^{\circ}$ & 11.93 \\
\hline 15 & $26.14489^{\circ}$ & $119.2840^{\circ}$ & $26.14491^{\circ}$ & $119.2840^{\circ}$ & 1.33 & $26.14489^{\circ}$ & $119.2840^{\circ}$ & 2.24 \\
\hline 16 & $26.14576^{\circ}$ & $119.287^{\circ}$ & $26.14573^{\circ}$ & $119.2870^{\circ}$ & 4.23 & $26.14570^{\circ}$ & $119.2870^{\circ}$ & 7.24 \\
\hline 17 & $26.14562^{\circ}$ & $119.2871^{\circ}$ & $26.14560^{\circ}$ & $119.2871^{\circ}$ & 4.83 & $26.14572^{\circ}$ & $119.2870^{\circ}$ & 12.05 \\
\hline 18 & $26.14543^{\circ}$ & $119.2872^{\circ}$ & $26.14542^{\circ}$ & $119.2872^{\circ}$ & 1.27 & $26.14535^{\circ}$ & $119.2872^{\circ}$ & 8.74 \\
\hline $\begin{array}{l}\text { 平均值 } \\
\text { Mean }(\mathrm{m})\end{array}$ & I & I & I & I & 2.40 & I & I & 6.02 \\
\hline
\end{tabular}

A
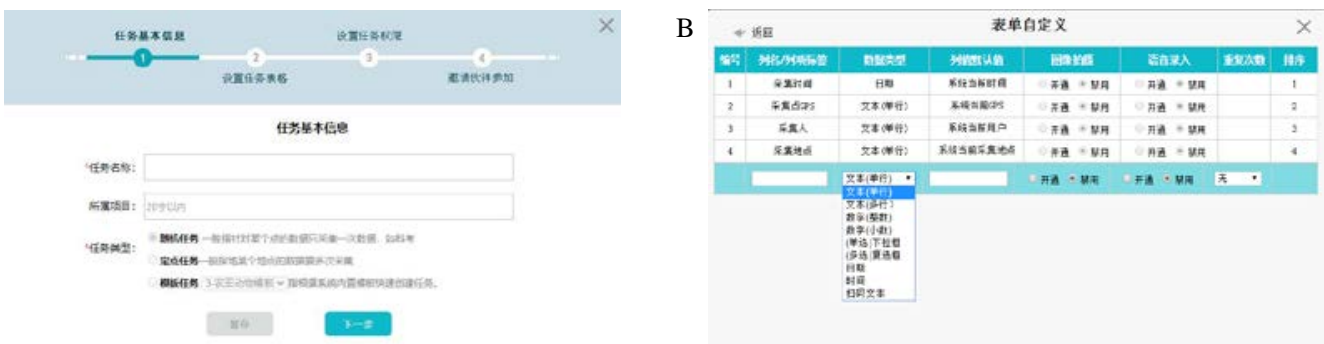

Mean (m)

Location captured by Jisibao A3S smartphone Location captured by Huawei P7 smartphone

$26.15058^{\circ}$

1.93

26.15062

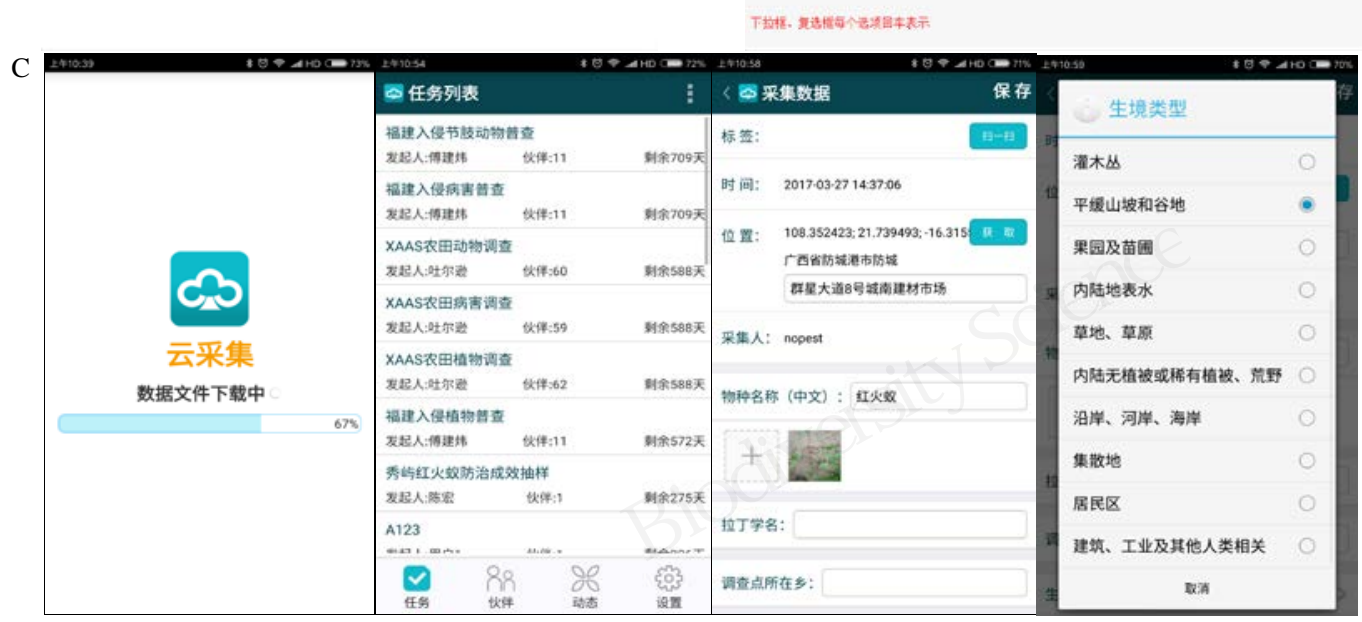

图4 云采集系统用户界面。A：任务创建界面截图; B: 表单自定义界面截图; C: 数据采集系统界面。

Fig. 4 User interface of the Yuncaiji data acquisitor. A: Screenshot of server-side creating a new task interface; B: Screenshot of server-side data customization interface; C: Screenshots of client-side data acquisition interface. 


\section{3 数据上传、查询与输出}

\subsection{1 数据上传}

移动终端采集的数据可以在网络环境下进行 批量上传。进入数据列表页面, 汶览已采集的数据 列表，可以获取采集时间、采集地址、采集人、入 侵生物名称和数据状态(分为未上传、上传中和已上 传), 点击右上角的 “上传”按钮, 进入数据批量上传 页面(图5), 将数据状态为“未上传”的采集记录及多 媒体文件打包上传到服务器端, 并将上传成功的采 集记录的数据状态标识为 “已上传”。数据上传过程 支持断点续传和多线程工作，不会因为网络中断而 发生数据丢失或损坏, 以保证数据传输速度。

\subsection{2 数据查询与输出}

采集的数据通过web服务器端管理软件进行授 权数据的在线查询，支持采集地点、采集时间、采 集人等关键词多条件组合查询。其中采集地址查询 模块采用 “省、市、县 3 级联动下拉框选项 + 乡镇村 详细地址关键词”组合查询; 采集时间查询模块采 用日期控件，按照日期起止区间进行过滤查询; 采 集人查询模块按人名进行精确查询(图6A)。当用户
需要对数据进行个性化处理时, 还可以将数据进行 XLS格式或KML格式的批量导出。XLS格式可以用 Microsoft Office和WPS Office打开并编辑, KML格 式可以被Google Earth和Google Maps识别并显示 (图6B)。

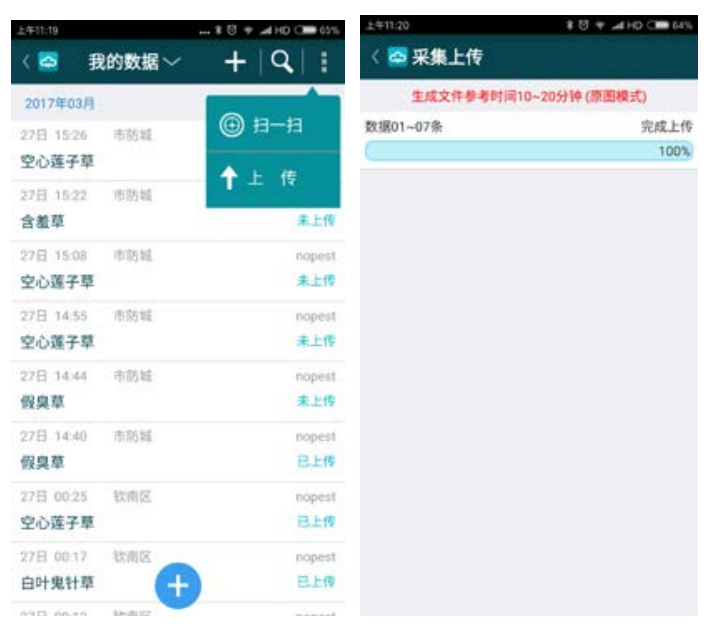

图5 云采集软件数据上传系统界面

Fig. 5 Screenshots showing a typical client-side end user data upload workflow of the Yuncaiji data acquisitor

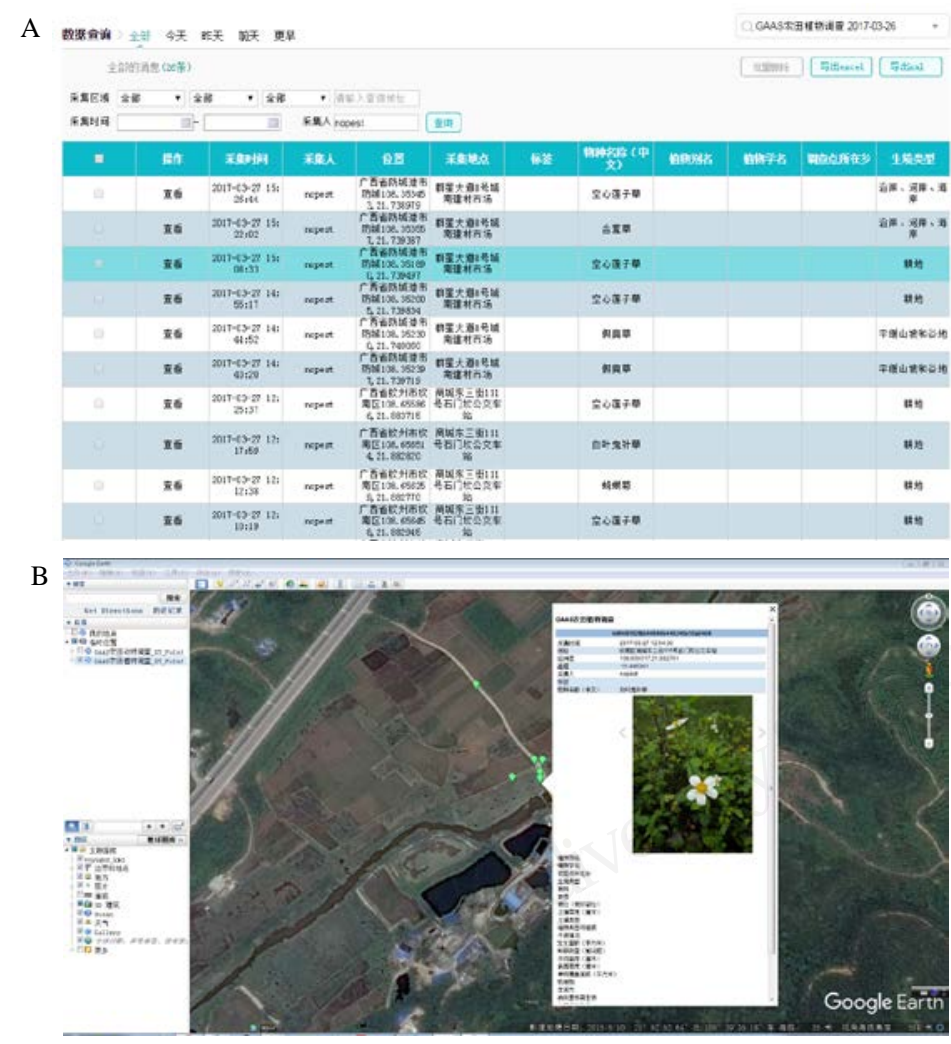

图6 云采集软件数据查询 $(\mathrm{A})$ 与导出数据在谷歌地球上的显示界面(B)

Fig. 6 Screenshot of the server-side query interface of the Yuncaiji data acquisitor (A) and a sample Google Maps image of a data collection item (B) 


\section{3 结论}

本研究开发的应用系统野外调查“云采集”软件 与同类系统 (申文明等, 2013; Köhl和 Gremmels, 2015; 黄国锐等, 2019)比较具有以下优点：(1)在任 务定制上, 设计了数据采集规范的自定义功能, 通 过将差异调查指标统一放到一个XML列的方法, 实现了在不修改数据库表结构的情况下, 就可以自 己定义数据采集规范, 添加任务采集人, 完成不同 应用需求的野外调查数据采集工作; (2)在数据录入 上, 设计了用户输入界面可定制的数据录入模式, 支持卫星导航定位自动获取、二维码扫描识别、下 拉框选择、拍照、录音等多种数据输入方式, 提高 了数据录入工作的效率。

据中国外来入侵物种数据库统计(冼晓青等, 2013), 我国入侵生物种类有500多种, 入侵生物扩 散大数据野外调查的数据表格有 3 类(动物、植物和 微生物) 24张, 包括调查地点信息表、问卷调查表、 物种排查表、普查表、植物样方表等。结合实际工 作需求, 依托国家重点研发项目、福建省科技重大 专项及福建省红火蚁疫情普查等项目的调查任务, 本研究建立的外来物种入侵大数据采集方法在福 建、广西、云南、新疆、北京等省份开展了应用试 验。截至2020年7月, 经过3年多的实际应用, 共发 起100多个入侵生物扩散大数据野外调查任务, 野 外调查“云采集”软件用户安装量 1,800 多人, 采集的 调查数据量达 250 多万条, 照片超过 370 GB。应用结 果表明, 利用Android移动终端进行入侵生物信息 采集与实时定位, 收集入侵物种信息, 实地拍摄物 种生境情况, 使户外数据采集从纸质记载变为精确 的数据库记录, 提高了数据统计分析的准确性, 实 现了定位、拍照、录入的一体化, 为中国外来入侵 物种地理分布信息系统(赵健等, 2016)提供了高质 量的监测数据。同时, 本文提出的技术方法同样适 用于其他生物多样性野外调查工作, 但是在调查规 划中需要根据不同的调查规程、对象和区域, 对分 析方法和指标作出相应的调整, 以满足不同调查的 需要。

\section{ORCID}

邱荣洲 (D) https://orcid.org/0000-0002-0905-8386 赵健 (D) https://orcid.org/0000-0003-2364-0687

\section{参考文献}

Chen B (2016) Geographic data management, refined route design and precise navigation in biodiversity field surveys. Biodiversity Science, 24, 701-708. (in Chinese with English abstract) [陈涁 (2016) 生物多样性野外调查地理信息管 理、路线精细设计和精确导航方法. 生物多样性, 24, 701708.]

Chen H, Xian XQ, Qiu RZ, Chi MX, Zhao J (2018) Planning and conducting field surveys of invasive alien species based on GIS. Biodiversity Science, 26, 44-52. (in Chinese with English abstract) [陈宏, 洗晓青, 邱荣洲, 池美香, 赵健 (2018) 基于 GIS 的外来入侵植物调查规划与外业实施. 生物多样性, 26, 44-52.]

Dai SQ, Zhao B (2016) Trends and challenges of ecosystem observations in the age of big data. Biodiversity Science, 24, 85-94. (in Chinese with English abstract) [戴圣骐, 赵斌 (2016) 大数据时代下的生态系统观测发展趋势与挑战. 生物多样性, 24, 85-94.]

Goodchild MF (2007) Citizens as sensors: The world of volunteered geography. GeoJournal, 69, 211-221.

Huang GR, Guo K, Wang SG, Jiang JB (2019) Design and implementation of handheld data acquisition terminal. Computer Science, 46, 567-569. (in Chinese with English abstract) [黄国锐, 郭康, 王世贵, 蒋金波 (2019) 一种手 持式数据采集终端的设计与实现, 计算机科学, 46, 567-569.]

Ju RT, Li H, Shih CJ, Li B (2012) Progress of biological invasions research in China over the last decade. Biodiversity Science, 20, 581-611. (in Chinese with English abstract) [鞠瑞亭, 李慧, 石正人, 李博 (2012) 近十年中 国生物入侵研究进展. 生物多样性, 20, 581-611.]

Köhl K, Gremmels J (2015) A software tool for the input and management of phenotypic data using personal digital assistants and other mobile devices. Plant Methods, 11, 25.

Luo QQ, Ju RT, Xu Y, Wang F (2011) Development and application of PDA pest real-time monitoring system of urban green space based on Web Service. Acta Phytophylacica Sinica, 38(1), 9-14. (in Chinese with English abstract) [罗卿权, 鞠瑞亭, 徐颖, 王凤 (2011) 基 于Web Service 的城市绿地有害生物PDA实时监控系统 的开发与应用. 植物保护学报, 38(1), 9-14.]

Millennium Ecosystem Assessment (2005) Ecosystems and Human Well-being: Synthesis. Island Press, Washington, DC.

Pan X, Zhang CJ, Wu L, Yan XQ (2020) Spatial-textal correlation analysis based on crowd source geospatial data. Geomatics and Information Science of Wuhan University, 45, 1910-1918. (in Chinese with English abstract) [潘晓, 张翠娟, 吴雷, 问晓倩 (2020) 众源地理空间数据的空间 文本相关性分析. 武汉大学学报 (信息科学版), 45, 1910-1918.]

Qiu RZ, Chen X, Chen H, Chi MX, Zhao J (2014) A data 
collection system for ecology and biodiversity investigation of Wuyi Mountain base on Android. Fujian Journal of Agricultural Sciences, 29, 188-193. (in Chinese with English abstract) [邱荣洲, 陈晓, 陈宏, 池美香, 赵健 (2014) 基于Android平台的武夷山生态与生物多样性野 外数据采集系统. 福建农业学报, 29, 188-193.]

Shan J, Qin K, Huang CQ, Hu XY, Yu Y, Hu QW, Lin ZY, Chen JP, Jia T (2014) Methods of crowd sourcing geographic data processing and analysis. Geomatics and Information Science of Wuhan University, 39, 390-396. (in Chinese with English abstract) [单杰, 秦昆, 黄长青, 胡翔 云, 余洋, 胡庆武, 林志勇, 陈江平, 贾涛 (2014) 众源 地理数据处理与分析方法探讨. 武汉大学学报(信息科学 版), 39, 390-396.]

Shen WM, Sun ZP, Zhang X, Chu D, Li F, Lü CB (2013) Research and implementation of mobile data collection system for field survey of ecological environment. Acta Ecologica Sinica, 33, 7846-7852. (in Chinese with English abstract) [申文明, 孙中平, 张雪, 初东, 李飞, 吕灿宾 (2013) 生态环境移动数据采集系统研究与实现. 生态学 报, 33, 7846-7852.]

Vankadavath RN, Hussain AJ, Bodanapu R, Kharshiing E, Basha PO, Gupta S, Sreelakshmi Y, Sharma R (2009) Computer aided data acquisition tool for high-throughput phenotyping of plant populations. Plant Methods, 5, 18.

Wan FH, Guo JY, Wang DH (2002) Alien invasive species in China: Their damages and management strategies. Biodiversity Science, 10, 119-125. (in Chinese with English abstract) [万方浩, 郭建英, 王德辉 (2002) 中国外来入侵
生物的危害与管理对策. 生物多样性, 10, 119-125.]

Xian XQ, Chen H, Zhao J, Wan FH (2013) Introduction to the database of invasive alien species in China. Plant Protection, 39(5), 103-109. (in Chinese with English abstract) [冼晓青, 陈宏, 赵健, 万方浩 (2013) 中国外来入侵物种数据库简 介. 植物保护, 39(5), 103-109.]

Zhao J, Chen H, Xian XQ, Qiu RZ, Wang ZH (2016) Development of Web GIS-based geographic distribution system on invasive alien species in China. Fujian Journal of Agricultural Sciences, 31, 663-667. (in Chinese with English abstract) [赵健, 陈宏, 冼晓青, 邱荣洲, 王宗华 (2016) 基于Web GIS的中国外来入侵物种地理分布信息 系统的设计与开发. 福建农业学报, 31, 663-667.]

Zhao QZ, Jin GC, Zhou WJ, Wang CJ, Dai JG (2015) Information collection system for diseases and pests in cotton field based on mobile GIS. Transactions of the Chinese Society of Agricultural Engineering, 31(4), 183-190. (in Chinese with English abstract) [赵庆展, 靳光 才, 周文杰, 汪传建, 戴建国 (2015) 基于移动GIS的棉 田病虫害信息采集系统. 农业工程学报, 31(4), 183-190.]

Zhou YC, Hu LL, Shen ZH, Yan BP (2008) Outdoor collection for scientific data based on PDA and its application. e-Science Technology \& Application, (1), 76-82. (in Chinese with English abstract) [周园春, 胡良霖, 沈志宏, 阎保平 (2008) 基于PDA的野外科考数据采集系统及其 应用. 科研信息化技术与应用, (1), 76-82.]

(责任编委: 万方浩 责任编辑: 黄祥忠)

\section{附录 Supplementary Material}

附录1 Web服务器端功能模块组织结构示意图

Appendix 1 Schematic diagram of the organizational structure of the web server module http://www.biodiversity-science.net/fileup/PDF/2021030-1.pdf

\section{附录2 手机客户端功能模块组织结构示意图}

Appendix 2 Schematic diagram of the organizational structure of the mobile client module http://www.biodiversity-science.net/fileup/PDF/2021030-2.pdf 
邱荣洲, 赵健, 陈宏, 冼晓青, 池美香, 翁启勇 (2021) 外来物种入侵大数据采集方法的建立与应用. 生物多样性, 29, 1377-1385. https://www.biodiversity-science.net/CN/10.17520/biods.2021030

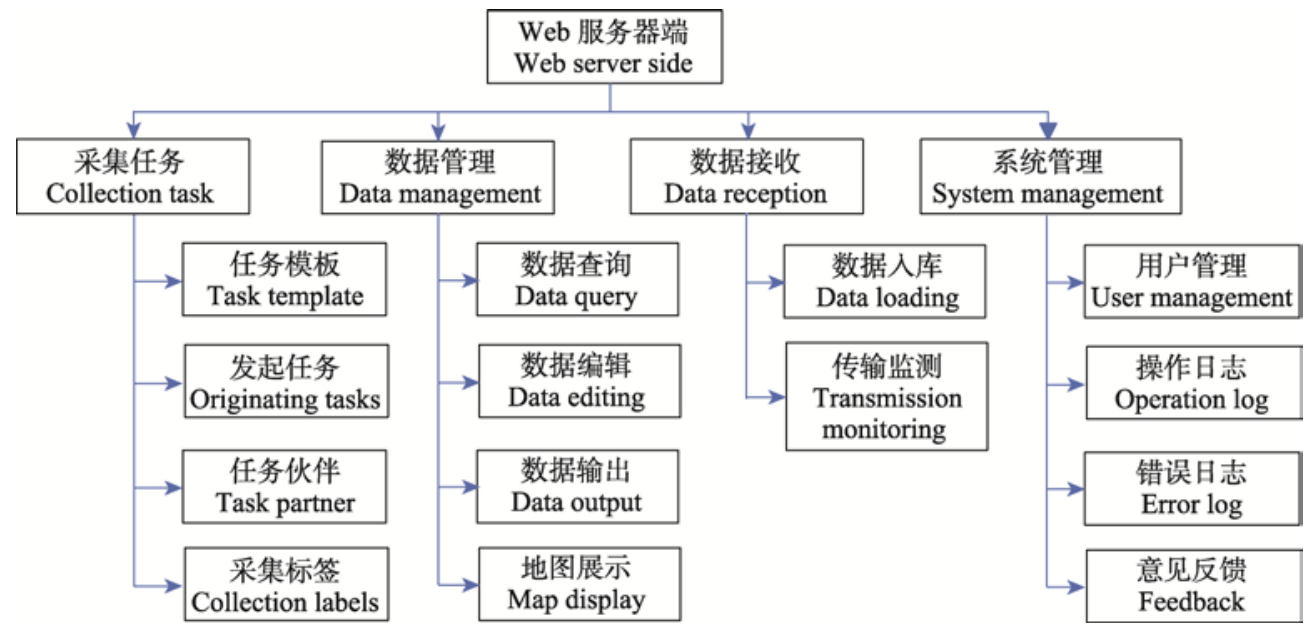

附录1 Web服务器端功能模块组织结构示意图

Appendix 1 Schematic diagram of the organizational structure of the web server module 


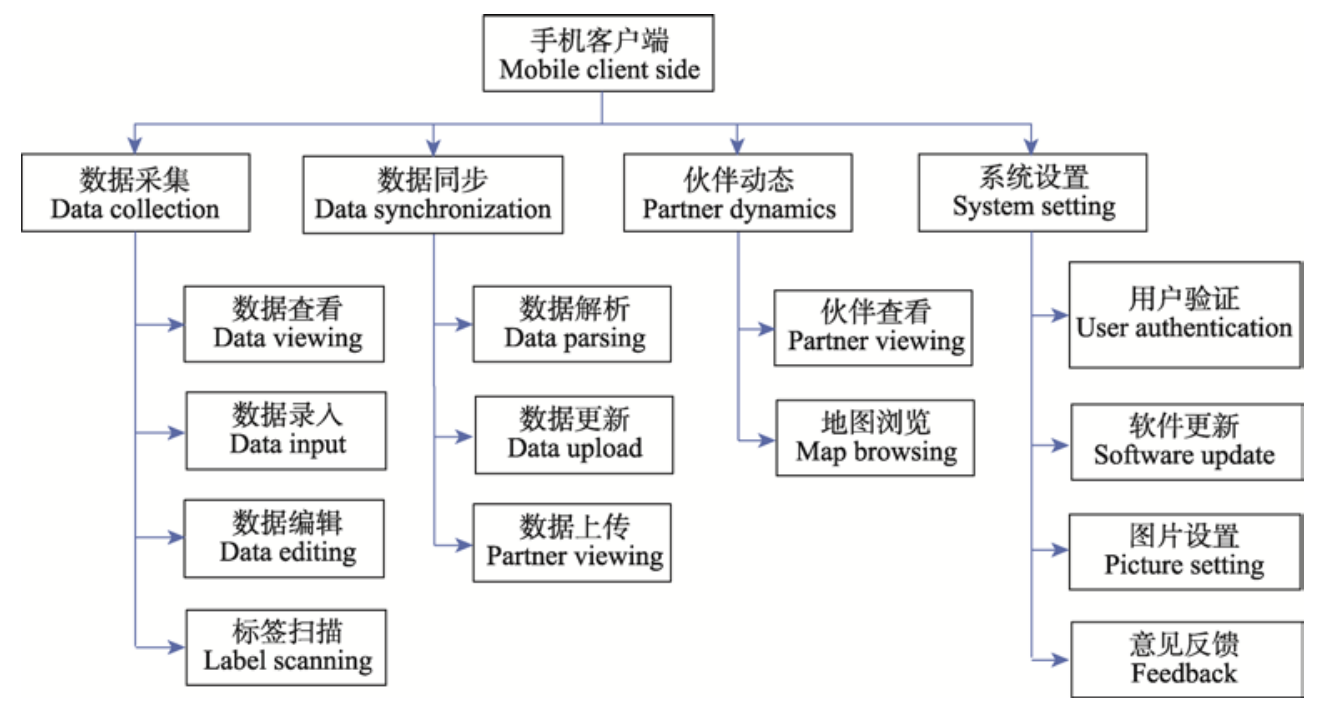

\section{附录2 手机客户端功能模块组织结构示意图}

Appendix 1 Schematic diagram of the organizational structure of the mobile client module 